\title{
Real-Time 4D Ultrasound Mosaicing and Visualization
}

\author{
Laura J. Brattain $^{1,2}$ and Robert D. Howe ${ }^{1}$ \\ ${ }^{1}$ Harvard School of Engineering and Applied Sciences, Cambrige, MA 02138 \\ ${ }^{2}$ MIT Lincoln Laboratory, 244 Wood St., Lexington, MA 02420 \\ \{brattain, howe\} aseas. harvard. edu
}

\begin{abstract}
Intra-cardiac 3D ultrasound imaging has enabled new minimally invasive procedures. Its narrow field of view, however, limits its efficacy in guiding beating heart procedures where geometrically complex and spatially extended moving anatomic structures are often involved. In this paper, we present a system that performs electrocardiograph gated 4D mosaicing and visualization of 3DUS volumes. Real-time operation is enabled by GPU implementation. The method is validated on phantom and porcine heart data.
\end{abstract}

Keywords: 3D ultrasound, electromagnetic tracking, graphic processing unit, volume mosaicing, volume registration.

\section{Introduction}

Beating heart procedures eliminate the need for cardiopulmonary bypass and its associated morbidities [1], and allow the surgeon to evaluate the procedure under physiologic loading conditions. Real-time 3D ultrasound (3DUS) imaging is often the imaging modality of choice for guiding these procedures. 3DUS is non-invasive, inexpensive, able to see through the blood, and can provide real-time diagnostic information of the anatomical region of interest. 3DUS also mitigates the difficulties in spatial perception associated with traditional 2DUS [2].

Intra-cardiac procedures often involve geometrically complex and spatially extended moving anatomic structures. Real-time visual feedback of the moving structures is critical to the success of these procedures. In order to realize the potential of 3DUS in guiding such procedures, two challenges must be addressed: high noise and narrow field of view. These limitations can be mitigated with a real-time panoramic view of the region of interest.

Electrocardiograph (ECG) gating is commonly used in the 4D reconstruction of a time-series of 3DUS volumes. Efforts have been reported in the reconstruction of gated 4DUS by mosaicing tracked 2DUS imaging [3][4][5]. In this case, 2DUS frames are acquired at the same point in the motion cycle of the target and composited into 3D volumes first, which then are assembled into a time series for sequential display.

With the arrival of real-time 3DUS machines, 3DUS volumes can be obtained directly and they offer improved spatial information compared to 2DUS, especially for geometrically complex targets with rapid motion. Brekke et al developed an algorithm that assembles ECG gated 3D cardiac ultrasound sub volumes into a symmetric pyramidal without using image registration [6]. They reported decreased 
geometric distortions compared to the non-stitched 3D volume. One major limitation of such a system is that it requires acquiring sub volumes at specific azimuth and elevation angles, and as a result, users have limited control over probe placement and orientation. Kutter et al presented a multi-modal 3DUS registration and mosaicing approach by incorporating information from co-registered CT [7]. However, CT data is not always available for clinical cases, especially for the non-intervention cases. In addition, pre-operative CT data usually does not accurately represent the real-time state of a patient's condition.

In this paper, we present the first system that performs freehand real-time 4DUS reconstruction and visualization based on streaming 3DUS data and ECG gating. The freehand capability is important as it allows clinicians to optimize the ultrasound acquisition geometry. Volume registration is performed through tracking the 3DUS probe using an electromagnetic (EM) tracking system. This method is similar to the reconstruction of 2D images as previously described, with a key difference being that the data throughput is one or two orders of magnitudes higher in 3D imaging. We address this issue by leveraging the parallel computing power of graphic cards. The computation of the volume mosaicing and rendering are done using a parallel implementation. In this paper, we begin with a description of the system setup, and then present our 4D reconstruction process, followed by results and discussions.

\section{System Configuration}

The system consists of three major hardware components: a GPU enabled computer (Dell Alienware Aurora, Intel Core i7 processor at 2.67GHz, 6GB RAM, NVIDIA GTX260 graphics cards), a 3DUS scanner (Philips SONOS 7500 with $x 4$ probe imaging at $8 \mathrm{~cm}$ depth and $28 \mathrm{~Hz}$ ) that can stream 3DUS images to the PC, and an EM tracking system (trackStar 3D Guidance System, Ascension Technology). The EM sensor is rigidly mounted to the 3DUS probe, and through a calibration process, we obtain the transformation between the ultrasound volume and the EM coordinates.

\subsection{Mosaicing Process}

The software application is implemented in $\mathrm{C} / \mathrm{C}++$, using multi-threading for data acquisition, CUDA (driver v.3.1.3.) for parallel computing, and OpenGL for visualization. At run time, in addition to the main thread, there are three threads running in parallel on the CPU: 3DUS volume streaming, EM tracking streaming, ECG waveform streaming. ECG phase time stamping is used for the acquisition of 3DUS volumes, which in turn are sent to the GPU, along with the EM tracking coordinates. The computations of volume registration, mosaicing and ray-casting are performed on the GPU. In order to perform mosaicing, we first calculate the transformation matrix between the input volume and the reference volume. The transformation matrix is determined by the EM tracker's outputs and the calibration matrix between the 3DUS coordinate system and the EM tracker's coordinate system. Fig. 1 illustrates the data flow between CPU and GPU.

Since the volume registration and compositing is a highly parallelizable process, we developed CUDA kernels to facilitate the computation. On the GPU, the transformation matrix is mapped to constant memory, and both the input and 
reference 3DUS data are mapped to CUDA 3D textures. Image registration uses trilinear interpolation, after which the registered volume and the reference volume are composited and the 3D texture that holds the reference frame is updated to the composite volume. There are a number of ways for compositing; we currently use the maximum intensity at the overlapping locations, because it eliminates the need to mask the void in the composite volume. To minimize computationally expensive memory transfer between host and device, the composite volume is sent to the raycasting kernel directly through device-to-device memory copy for rendering.

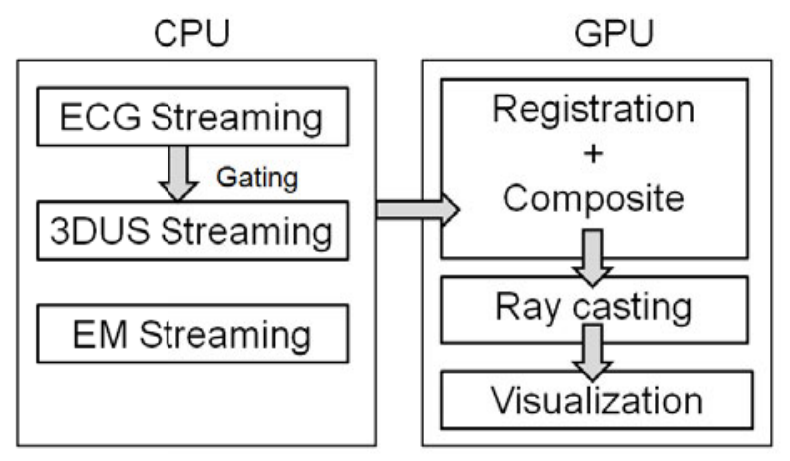

Fig. 1. Data flow between CPU and GPU

\subsection{ECG Gating}

Cardiac synchronization limits artifacts due to motion of the heart, thus enabling clear visualization across the phases of the cardiac cycle. The ECG $\mathrm{R}$ wave peak is typically used for this purpose as it can be readily identified in the ECG signal. The interval between $\mathrm{R}$ wave peaks, the RR interval, defines one cardiac cycle for gating. In our study, we perform $\mathrm{R}$ wave peak detection in real-time and divide an $\mathrm{RR}$ interval into a user-specified number $N$ of equally spaced bins.

The user first chooses a location to image and sets it as the reference frame. $N$ bins of gated data are collected at this location and saved as the reference volumes. The subsequent volumes acquired are added to the final mosaiced volume by being registered into the corresponding reference volume with the matching bin number, as above. To acquire these volumes, the user moves the probe to a desired location and triggers the data acquisition at that location. The mosaicing is performed immediately and can be visualized at real time. The final time-series consists of $N$ mosaiced volumes. Fig. 2 illustrates this process.

\section{Experimental Validation}

\subsection{Methods and Results}

We use phantom data in a water tank to develop and test the system. The phantom consists of horizontal, vertical and diagonal strings suspended across a rigid frame 
with a dimension of $8 \times 6 \mathrm{~cm}$ (Fig. 3A). A string phantom provides an easy means to validate the mosaicing result by confirming the mosaiced colinearity of the segments from each input volume. The phantom is suspended in the water tank with one side connected rigidly to a cam device. The cam device simulates the cyclic motion of the mitral annulus (Fig. 3B). A signal from the cam device simulates the output of an ECG monitor. Fig. 4 (A) shows one mosaiced result of the string phantom, and (B) (E) are four of the 3DUS volumes used in the creation of the mosaiced volume. It is evident that the mosaiced volume provides a wider field of view. The input volume has a dimension of $128 \times 48 \times 208$ voxels, and the dimension of the mosaiced volume is $320 \times 144 \times 210$ voxels. On average, the time used to perform every two volume registration and stitching on the GPU is $25 \mathrm{~ms}$ on the system described above. The time to mosaic 8 bins is $175 \mathrm{~ms}$. This mosaicing process is applied to each bin of the motion cycle (Fig. 5).

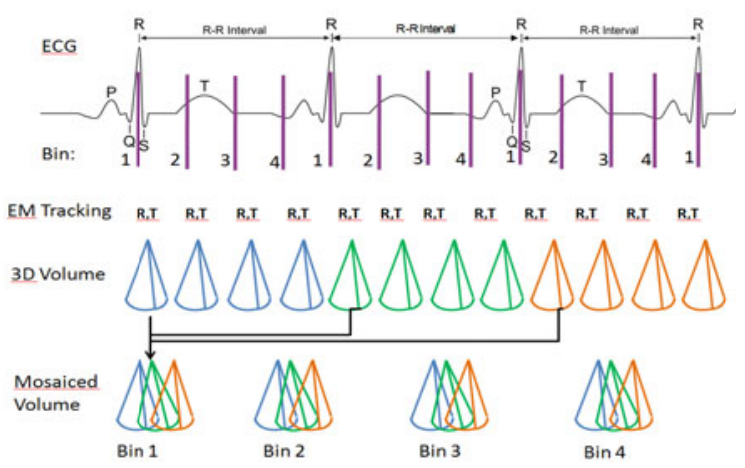

Fig. 2. 4DUS data mosaicing with ECG gating
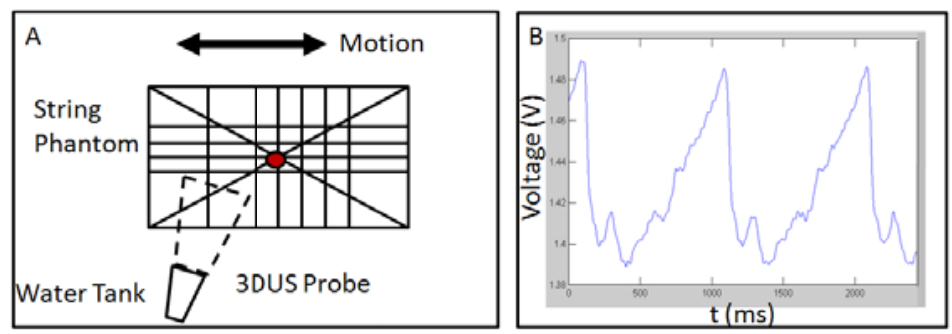

Fig. 3. Experiment setup. (A) Water tank with string phantom. The red dot indicates a fiducial point in subsequent figures. (B) Periodic motion trajectory.

We then conducted experiments using a porcine heart in a water tank. As with the string phantom, the heart is mounted on a plastic frame which is rigidly connected to the cam device. The heart (Fig. 6) is positioned sideways, with the top and bottom portions sectioned and removed, allowing water to flow through the heart, which 


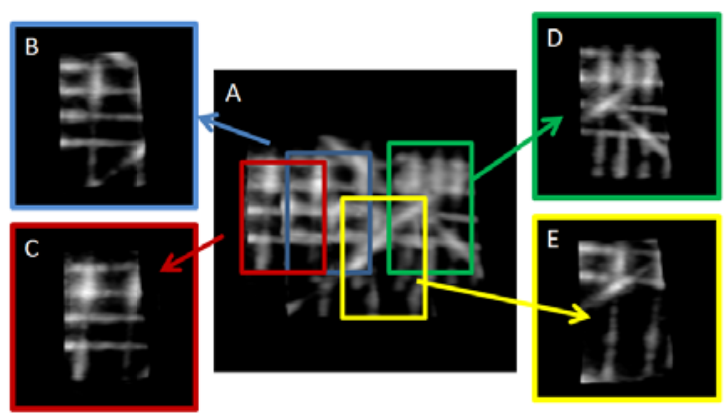

Fig. 4. (A) One mosaiced result. (B)-(E) Four of the input volumes.
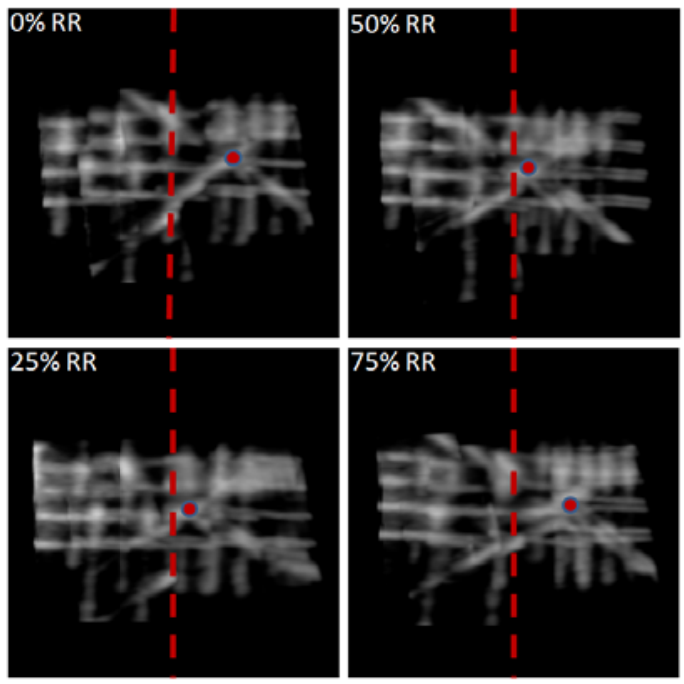

Fig. 5. Mosaiced results at different RR intervals. The red dot is the fiducial point on the string phantom. The red dotted line indicates the mid line of the cross section. The varying distance between the fiducial point and the mid line is the result of the motion of the phantom.

causes the valves to open and close periodically. The ultrasound probe is positioned adjacent to the heart at the region where the right atrium and tricuspid valve are located (Fig. 6 (D)).

Fig. 6 (E) is the result of mosaicing 18 3DUS volumes at each of $N=8$ time bins, using a total of 144 volumes. The red arrow indicates the tricuspid valve, which is open at $0 \% \mathrm{RR}$ interval and closed at $50 \% \mathrm{RR}$ interval. This periodic motion can be seen clearly in the visualization loop of the complete time series. This highlights the value of the visual feedback for real-time intra-cardiac procedural guidance. 


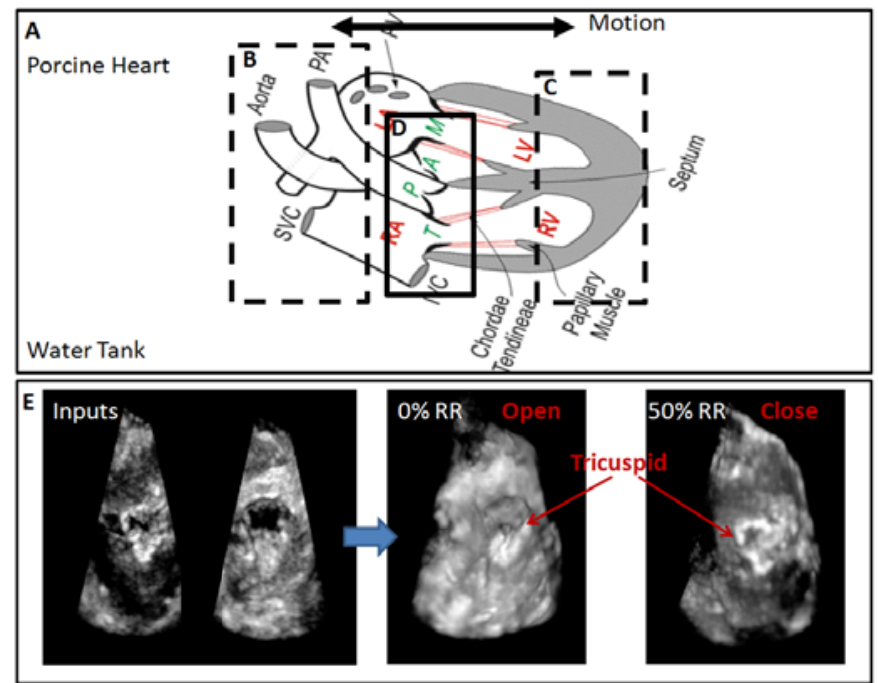

Fig. 6. (A) Porcine heart [8]. The sectioned portion $\mathrm{B}$ and $\mathrm{C}$ are removed. (D) Imaging section. (E) Two Input volumes (left) Mosaicing results at $0 \%$ and 50\% RR interval (right).

\subsection{Validation}

We use the string phantom to validate the co-linearity of the mosaiced line segments. For a mosaiced line, we first identity the line segment from the reference frame since it is the segment that the other segments are registered to. This line segment is used as the centroid of the mosaiced line. We then calculate the number of non-trivial voxels around the centroid at a given radius. We expect to see that most of the contributing voxels are around the centroid, and the number of contributing voxels falls off quickly when the radius increases. Fig. 7 is a plot of the percentage of the contributing voxels vs. radius from the centroid. The width of the mosaiced line is roughly 4 voxels, which equals the width of the line segment in the reference frame.

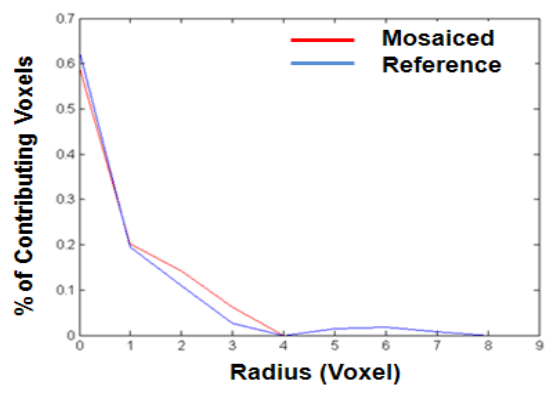

Fig. 7. Co-linearity of mosaiced line segments 


\section{Discussion}

We have developed the first real-time freehand 4D mosaicing and visualization system using ECG gated 3DUS streaming data. 3DUS streaming provides a much higher data rate than 2DUS based 3D reconstruction. This allows faster updates of the mosaiced image, but requires parallel computation. Although present 3DUS machines have a lower spatial resolution than the best 2DUS machines, 3DUS resolution is improving rapidly. For example, newer machines (e.g. the Philips iE33 scanner) have 2-3 times the spatial resolution of the machine used in this study.

\subsection{Temporal Resolution and Imaging Time}

There is a tradeoff between imaging time to build or update the mosaic and time resolution (i.e. the number of time bins $N$ ). Temporal resolution is particularly important for accurately capturing the motion of fast-moving structures such as cardiac valves. For example, the peak velocity of the mitral valve annulus has been estimated as $v=210 \mathrm{~mm} / \mathrm{s}$ [9], so assuming a typical RR interval of $T_{R R}=1 \mathrm{~s}$ and with number of bins $N=10$, the spatial blur within one time bin can be as high as $d x=v\left(T_{R R} / N\right)=21 \mathrm{~mm}$. This shows that high temporal resolution reduces the effect of spatial blurring.

On the other hand, higher temporal resolution will result in longer data acquisition and processing time and higher demand on computer memory size. In addition, the current 3DUS streaming is at approximately $30 \mathrm{~Hz}$, which limits the data acquisition to 30 volumes per second.

\subsection{Real-Time Overlay}

One of the important features that this system offers is a "big picture" view of a large section of the heart, combined with a "fovea" real-time view of the smaller region where the procedure is performed. The mosaiced time series can be viewed as a map in the background, which can be extremely useful to the user for navigating to a specific location and for planning subsequent surgical steps. The specific location at which the catheter or instrument is working is then automatically overlayed on the mosaiced times series in real-time. Any region of the mosaic can be updated by simply moving the probe to view that region, and the real-time view follows the instrumentation. Color coding of real-time versus previously mosaiced data can inform the clinician of which regions represent the current versus historical images. This allows for simple and reliable control of the image acquisition and display process.

\section{Conclusion}

In this paper, we presented a system for real-time 4DUS mosaicing and visualization. The system integrates EM tracking systems and GPU implementation for real-time registration and mosaicing of high data rate 3DUS images. ECG time stamping provides synchronization with heart motion. Temporal resolution can be controlled by 
the user, and real-time data is continuously overlayed on the mosaic. The system merges previously-acquired wide-area data with real-time focal data, facilitating navigation and procedure execution with a simple user interface.

Acknowledgments. The Harvard University portion of the work is sponsored by US National Institutes of Health under grant NIH R01 HL073647-01. The MIT Lincoln Laboratory portion of the work is sponsored by the Department of the Air Force under Air Force contract \#FA8721-05-C-0002. Opinions, interpretations, conclusions and recommendations are those of the author and are not necessarily endorsed by the United States Government.

\section{References}

1. Murkin, J.M., Boyd, W.D., Ganapathy, S., Adams, S.J., Peterson, R.C.: Beating Heart Surgery: Why Expect Less Central Nervous System Morbidity? Annals of Thoracic Surgery 68, 1498-1501 (1999)

2. Cannon, J.W., Stoll, J.A., Salgo, I.S., Knowles, H.B., Howe, R.D., Dupont, P.E., Marx, G.R., del Nido, P.J.: Real-time three dimensional ultrasound for guiding surgical tasks. Computer Aided Surgery 8(2), 82-90 (2003)

3. Treece, G.M., Prager, R.W., Gee, A.H., Cash, C.J.C., Berman, L.: Grey-scale gating for freehand 3D ultrasound. In: IEEE. Int. Symp. on Biomedical Imaging (2002)

4. Pieper, S., Halle, M., Kikinis, R.: 3D Slicer. In: IEEE Int. Symp. on Biomedical Imaging (2004)

5. Pace, D., Gobbi, D., Wedlake, C., Gumprecht, J., Boisvert, J., Tokuda, J., Hata, N., Peters, T.: An open-source real-time ultrasound reconstruction system for four-dimensional imaging of moving organs. In: Systems and Architectures for Computer Assisted Interventions. MICCAI 2008 Workshop (2008)

6. Brekke, S., Rabben, S.I., Stoylen, A., Haugen, A., Haugen, G.U., Steen, E.N., Torp, H.: Volume Stitching in Three-Dimensional Echocardiography: Distortion Analysis and Extension to Real Time. Ultrasound in Medicine and Biology 33, 782-796 (2007)

7. Kutter, O., Wein, W., Navab, N.: Multi-modal Registration Based Ultrasound Mosaicing. In: Yang, G.-Z., Hawkes, D., Rueckert, D., Noble, A., Taylor, C. (eds.) MICCAI 2009. LNCS, vol. 5761, pp. 763-770. Springer, Heidelberg (2009)

8. CV Physiology,

http: / /www. cvphysiology.com/Heart\%20Disease/HD001.htm

9. Kettler, D.T., Plowes, R.D., Novotny, P.M., Vasilyev, N.V., del Nido, P.J., Howe, R.D.: An Active Motion Compensation Instrument for Beating Heart Mitral Valve Surgery. In: IEEE Intl. Conf. Intelligent Robots \& Systems (2007) 